IdeAs

Idées d'Amériques

16 | 2020

Les marges créatrices : intellectuel.le.s afrodescendant.e.s et indigènes auX Amériques, XIX-XXe siècle

\title{
La présidence Trump et les contre-pouvoirs institutionnels : une revanche du Watergate?
}

\section{Corentin Sellin}

\section{OpenEdition}

Journals

Édition électronique

URL : http://journals.openedition.org/ideas/9627

DOI : $10.4000 /$ ideas. 9627

ISSN : $1950-570$

Éditeur

Institut des Amériques

Référence électronique

Corentin Sellin, «La présidence Trump et les contre-pouvoirs institutionnels : une revanche du

Watergate? », IdeAs [En ligne], 16 | 2020, mis en ligne le 01 octobre 2020, consulté le 10 décembre 2020. URL : http://journals.openedition.org/ideas/9627 ; DOI : https://doi.org/10.4000/ideas.9627

Ce document a été généré automatiquement le 10 décembre 2020.

\section{$(1) \Theta$}

IdeAs - Idées d'Amériques est mis à disposition selon les termes de la licence Creative Commons Attribution - Pas d'Utilisation Commerciale - Pas de Modification 4.0 International. 


\title{
La présidence Trump et les contre- pouvoirs institutionnels : une revanche du Watergate?
}

\author{
Corentin Sellin
}

1 Le $1^{\text {er }}$ mai 1974, devant le tribunal fédéral du district de D.C et le juge John Sirica, l'avocat James St Clair déclarait comme défense liminaire du président Nixon, assigné par le procureur spécial Jaworski pour des bandes d'enregistrement relatives au cambriolage du Watergate : "Le président me demande de plaider qu'il est un monarque aussi puissant que Louis XIV mais pour un mandat de quatre ans seulement, et qu'il n'est justiciable devant aucun tribunal de ce pays sauf celui de l'impeachment $»^{1}$. Cette phrase, restée célèbre, rappelle qu'au-delà des enjeux particuliers du Watergate, la période 1973-1974 fut celle d'une tension inédite pour les institutions étasuniennes.

2 Richard Nixon entendait mener une présidence (pouvoir exécutif) délivrée des contrepouvoirs (checks and balances) prévus par la Constitution de 1787, au premier rang desquels le Congrès (pouvoir législatif) et les tribunaux (pouvoir judiciaire). L'affrontement de Richard Nixon avec les tribunaux est bien documenté dans le cas du Watergate, où il essaie de faire prévaloir à partir du printemps 1973 la notion de privilège de l'exécutif pour ne pas avoir à se soumettre aux actes de procédure des procureurs spéciaux et remettre les bandes d'enregistrement de la Maison Blanche pouvant l'incriminer. Mais l'ampleur du scandale du Watergate masque souvent la guerre menée au Congrès par Nixon pour bloquer certaines dépenses autorisées par le vote des chambres législatives que le président désapprouvait politiquement. Cette pratique d'impoundment (retenue) par Nixon s'était caractérisée depuis 1972 par son ordre à l'administrateur de l'agence de protection de l'environnement (EPA) de ne pas distribuer l'intégralité des fonds alloués aux États par les amendements à la loi sur le contrôle de la pollution des eaux. Nixon avait apposé son veto, outil constitutionnel de son contrôle des pouvoirs du Congrès, sur ces amendements mais les deux chambres l'avaient surmonté à la majorité des deux tiers. La retenue des fonds, si elle avait déjà 
été utilisée par d'autres présidents pour des motifs techniques, apparaissait donc là comme un veto politique déguisé, sans aucun fondement constitutionnel.

Dans cette confrontation, comme celle du Watergate avec les tribunaux, la Cour Suprême avait fini par délimiter les rapports de force institutionnels entre présidence et contre-pouvoirs. Le fameux arrêt USA $v$ Nixon du 24 juillet $1974^{2}$, loin d'être une défaite pour la présidence puisque reconnaissant pour la première fois l'existence implicite d'un privilège de l'exécutif dans l'article II de la Constitution, ne le jugeait pas suffisant pour contrevenir à l'égalité de droit dans une procédure criminelle et obligeait le président Nixon à remettre les bandes assignées au procureur. Un autre arrêt de la Cour Suprême, moins connu parce que plus tardif et postérieur à la démission de Nixon, Train $v$ city of New York, en février 1975, réaffirmait le pouvoir exclusif du Congrès, dit « pouvoir du porte-monnaie » (power of the purse) à allouer les ressources budgétaires et fixer leur montant ${ }^{3}$.

4 Ces arrêts de la Cour Suprême n'étaient que le début d'une véritable reprise en main par le Congrès de la présidence, visant à réformer tous les abus de pouvoir constatés à l'ère Nixon et à en éviter la reproduction future. Or, pour l'instant, la présidence Trump a constitué une offensive constante et méthodique pour démanteler tous ces garde-fous de la période post-Watergate contre l'expansion des pouvoirs présidentiels.

Dominé durant ses deux premières années par l'enquête du procureur spécial Mueller sur l'éventuelle collusion de la campagne présidentielle Trump avec la Russie, ce mandat a d'abord remis en jeu le statut pénal du président des États-Unis, encadré par la jurisprudence USA $v$ Nixon de 1974 et le rapport hiérarchique des procureurs fédéraux à l'exécutif. En 1978, le Congrès à majorité démocrate dans ses deux chambres, et encouragé par le président réformateur Carter, avait voté l'Ethics in Government Act, loi dont le titre VI imposait au ministre de la Justice de demander à un panel de trois juges de la Cour d'Appel de Washington D.C. l'installation d'un procureur spécial (puis indépendant) si son enquête préliminaire démontrait la nécessité d'investigations supplémentaires contre toute personne exerçant des responsabilités dans l'exécutif (ou dans une campagne présidentielle majeure). Cette loi, baptisée dans ses prémices parlementaires en 1975-76 le Watergate Reform and Reorganization Act, «sortait » ainsi toute enquête à venir sur la présidence de la chaîne hiérarchique de l'exécutif et du ministre de la Justice. Cela devait éviter l'extrémité atteinte sous Nixon lorsqu'il avait renvoyé le premier procureur spécial Archibald Cox, ainsi que deux ministres de la Justice (attorney general), Richardson et Ruckelshaus qui refusaient de le démettre, lors de la fameuse journée du 20 octobre 1973, dite du Massacre du Samedi Soir 4 . Mais ce titre VI de la loi de 1978 sur le statut des procureurs indépendants était non définitif et renouvelable tous les cinq ans. Après 20 ans de guérilla incessante des républicains, l'enquête du procureur spécial Starr contre Bill clinton fit basculer une majorité d'élus démocrates contre elle, reprochant aux procureurs indépendants leurs pouvoirs illimités et sans contrôle de coût. En juin 1999, le statut du procureur indépendant n'était pas renouvelé et le ministre de la Justice, nommé par le président, récupérait seul l'autorité de nomination d'un procureur spécial, selon des modalités désormais définies par un règlement interne à son département ${ }^{5}$. Le ministre de la Justice devrait évaluer le risque de conflit d'intérêt en cas d'enquête à mener sur un détenteur du pouvoir exécutif avant de nommer un procureur spécial qui resterait soumis à son autorité pour toute décision de poursuite. Autrement dit, le consensus du Watergate sur un président devant être soumis à une justice criminelle indépendante 
ne tenait plus qu'à la bonne volonté des acteurs institutionnels et Donald Trump a précisément dévoilé la fragilité de cette nouvelle construction juridique.

D'une part, conformément à la doctrine conservatrice dite de l'exécutif unitaire formulée dans l'administration Reagan en réaction aux limites imposées à la présidence après le Watergate, le président Trump a toujours affirmé considérer le ministre de la Justice et son département comme ses subordonnés directs, devant lui obéir, y compris pour des enquêtes à but politique. Avec la verdeur habituelle de son vocabulaire, D. Trump affirma ainsi dans un entretien au New York Times en décembre 2017 : «J'ai le droit absolu de faire ce que je veux au ministère de la Justice $»^{6}$. Cela explique ainsi sa décision en mai 2017 de renvoyer James Comey, le patron du FBI, agence fédérale administrée par ministère de la Justice. Trump concéda par la suite, avant de se dédire, que ce renvoi tenait à l'enquête que Comey dirigeait alors sur les liens éventuels entre campagne Trump et Russie ${ }^{7}$. Ce choix abrupt, en complète rupture avec l'équilibre des pouvoirs post-Watergate, retrouvait pleinement la logique du Massacre du Samedi Soir de Nixon. En parfaite adéquation, au contraire, avec les obligations imposées depuis Watergate, le ministre de la Justice de Trump, Jeff Sessions, s'était déjà récusé depuis mars 2017 de son autorité sur cette enquête du FBI puisque susceptible d'être interrogé comme témoin. Cette récusation amenait son adjoint Rod Rosenstein, désormais en responsabilité, à nommer le procureur spécial Robert Mueller le 11 mai 2017 pour reprendre l'enquête du FBI sur le Russiagate. Mais les quolibets publics, voire les injures, du président Trump à son ministre de la Justice Sessions, dénotaient bien la confrontation de deux logiques antagonistes à l'intérieur de l'administration.

7 D'autre part, quand Donald Trump finit par renvoyer Sessions, sans cacher là aussi qu'il le faisait à cause de sa récusation ${ }^{8}$, et alors que le procureur spécial Mueller entrait dans les dernières étapes de son enquête, il installa William Barr comme nouvel attorney general en janvier 2019 avec l'assentiment de la majorité républicaine au Sénat. En Barr, Trump promouvait un homme qu'il avait d'abord pressenti pour être son avocat de la défense, démontrant la conception qu'il avait du rôle institutionnel de son ministre de la Justice. Il choisissait aussi l'un des principaux théoriciens de l'exécutif unitaire selon lequel les poursuites judiciaires, décidées par le ministère de la Justice, branche de l'exécutif, relèvent pleinement du domaine présidentiel défini à l'article II de la Constitution. Lors de son premier passage au ministère de la Justice entre 1989 et 1992, dans l'administration Bush Sr, comme directeur du bureau de conseil juridique puis ministre, William Barr avait ainsi rédigé de nombreux mémos pour remettre en cause les limitations du pouvoir présidentiel par le Congrès, comme par exemple sur la guerre du Golfe qu'il estimait pouvoir être menée sans approbation du Congrès.

Une fois rappelé comme ministre par Trump pour superviser l'enquête Mueller, Barr s'acquitta avec le procureur spécial Mueller de la mission confiée. Il décida fin mars 2019, grâce à l'obligation faite au procureur spécial de lui remettre son rapport avant publication et de discuter des éventuelles décisions de poursuite, de rendre public son "résumé » du rapport. Cette synthèse de Barr, expédiée le 24 mars aux comités judiciaires du Congrès, déchargeait le président de tout soupçon de collusion avec la Russie et d'entrave à la justice ${ }^{9}$. Or, quelques semaines plus tard, le rapport Mueller, publié avec de larges coupes ordonnées par le ministre de la Justice, concluait pourtant ne pas pouvoir exonérer le président de l'entrave à la justice ${ }^{10}$. On apprenait aussi que Mueller, procureur spécial obéissant aux réglementations et normes du ministère de la Justice, avait de toute façon renoncé par avance à poursuivre le président en justice. Il 
se conformait à deux mémos internes du bureau de conseil juridique du ministère de 1973 et 2000, jamais éprouvés devant les tribunaux mais concluant à l'immunité de poursuite pénale d'un président en exercice ${ }^{11}$. Si les formes d'une enquête judiciaire indépendante sur le président, héritées du Watergate, avaient été préservées, D. Trump, par une obstruction permanente - qu'on en juge par les centaines de tweets publics faisant pression sur Mueller - et une soumission politique du ministère de la Justice, en avait court-circuité en large partie les modalités pratiques.

travail de sape des réformes du consensus post-Watergate a pu être observé lors de l'affaire dite ukrainienne. À cette occasion, la bataille principale a opposé le président au Congrès, préoccupé à plusieurs titres par l'appel le 25 juillet 2019 de D. Trump au président ukrainien Zelensky. Durant ce dernier, il avait demandé à celui-ci de reprendre les enquêtes concernant le fils de son principal rival démocrate et exvice-président Joe Biden. Cette demande intervenait de surcroît alors que le président Trump avait suspendu à la fin juin 2019 une aide militaire de presque 400 millions de dollars à l'Ukraine pourtant votée par le Congrès, pouvant laisser supposer un quid pro quo : « aide militaire en échange d'une enquête sur le fils Biden ».

Cet appel avait été rapporté au Congrès par la procédure du whistleblowing (lancement d'alerte) codifiée à l'intérieur de la communauté du renseignement, plusieurs officiers du conseil de sécurité nationale ayant été choqués par la politique menée vis-à-vis de l'Ukraine et le contenu de l'appel du 25 juillet. L'alerte fut transmise par l'inspecteur général du renseignement Atkinson, mettant en avant une fonction créée par une autre loi post-Watergate, l'Inspector General Act de $1978^{12}$. Ces inspecteurs généraux étaient alors créés pour établir dans les départements exécutifs des contrôleurs indépendants pouvant rendre compte au Congrès de tout abus de pouvoir ou dysfonctionnement grâce, entre autres, aux lanceurs d'alerte. Néanmoins, afin d'assurer un soutien bipartisan à cette institution nouvelle, il fut décidé d'en laisser la nomination - et donc la révocation - au président, avec confirmation par le Sénat. L'inspecteur général Atkinson fut d'ailleurs installé par le président Trump et la majorité républicaine du Sénat en mai 2018. L'indépendance des inspecteurs généraux ne tenant qu'à leur lien fonctionnel avec le Congrès, celle-ci avait été renforcée en 2008, une nouvelle loi du Congrès obligeant le président à justifier au Congrès une décision de renvoi d'un inspecteur général avec un préavis de trente jours ${ }^{13}$.

11 La transmission du lancement d'alerte par Atkinson auprès du Congrès déclencha une enquête puis une mise en accusation (impeachment) par la Chambre à majorité démocrate à l'automne 2019, reprenant là aussi le modèle du Watergate puisque Richard Nixon avait été soumis à la même procédure constitutionnelle entre mai et juillet 1974. Mais la suite de la procédure contre Trump fit mesurer l'étendue de son renversement des normes du Watergate quant aux comptes à rendre par la présidence. Alors que les actes d'accusation en impeachment contre Nixon avaient été votés fin juillet 1974 au comité judiciaire par une partie des élus républicains, aucune voix de la minorité républicaine à la Chambre ne manqua à Donald Trump contre sa mise en accusation fin 2019, lors du vote en comité ou en assemblée plénière. Et fort de ce soutien uniforme du parti, quand ce dernier avait lâché Nixon début août 1974 le contraignant à la démission, Trump put affronter sans coup férir le procès en destitution au Sénat avec sa majorité de 53 Républicains. Acquitté des deux chefs d'accusation d'abus de pouvoir et d'obstruction au Congrès le 5 février 2020, Trump a 
pu accentuer son démantèlement des règles de contrôle de la présidence imposées depuis le Watergate.

Le 5 avril 2020, le président Trump limogea l'inspecteur général Atkinson, arguant avoir perdu confiance en lui et lui reprochant explicitement sa transmission du lancement d'alerte au Congrès. Ce renvoi se faisait, de surcroît, par un congé immédiat, sans respecter le délai de 30 jours de préavis au Congrès. Après Atkinson, Trump a renvoyé quatre autres inspecteurs généraux en avril-mai, dont Steve Linick, celui du département d'État qui avait entamé une enquête administrative sur les pratiques du ministre Mike Pompeo. A chaque fois, le président Trump invoqua le besoin de loyauté à sa personne, ce qui apparaît incompatible avec la fonction même de contrôle impartial des inspecteurs généraux. Donald Trump n'est cependant pas le premier président républicain à procéder ainsi : en 1981, Ronald Reagan avait renvoyé tous les inspecteurs généraux en place lors de son accession au pouvoir. Mais la pression des deux chambres du Congrès, pourtant à majorité républicaine, le força à renoncer en partie et à maintenir cinq des seize inspecteurs généraux. Il n'en fut rien, faute de consensus entre les deux chambres, pour le Congrès face à Trump.

Cette rapide comparaison historique met en évidence la différence fondamentale entre Trump et Nixon dans la dynamique relationnelle entre présidence et contre-pouvoirs. Nixon fut contraint, comme lorsqu'il laissa le ministre de la Justice par intérim Bork renommer un procureur spécial après le Massacre du Samedi Soir, par un Congrès à majorité démocrate dans les deux chambres. Trump a, lui, toujours bénéficié d'une majorité solide au Sénat. Et cette majorité est surtout le reflet d'une polarisation extrême des deux formations républicaine et démocrate. Alors que Nixon fut mis en accusation par une coalition d'élus centristes des deux camps à la Chambre réunie autour du républicain Tom Railsback ${ }^{14}$, ce dépassement institutionnel des clivages politiques est devenu impossible à l'ère Trump. Une preuve flagrante en a été fournie lors de son procès en impeachment au Sénat en janvier 2020 lorsque l'organe de contrôle indépendant du Congrès, le Government Accountability office (GAO) a conclu que la présidence Trump, en bloquant l'aide militaire à l'Ukraine, avait violé une autre loi consécutive au Watergate et aux agissements de Nixon, l'Impoundment Control Act de juillet $1974^{15}$. A l'époque, cette loi avait été adoptée à la quasi-unanimité par démocrates et républicains, car il s'agissait de défendre le "pouvoir du portemonnaie » de l'institution parlementaire face aux retenues de la présidence. En 2020, le patron républicain du comité budgétaire du Sénat, Richard Shelby, eut comme seule réaction: «Ce n'est pas au GAO de déterminer qui enfreint la loi.» Comment mieux caractériser l'abandon par les élus républicains de l'idéal post-Watergate d'une présidence limitée par les contre-pouvoirs au profit d'un blanc-seing accordé à Donald Trump?

\section{NOTES}

1. Cité dans Richard Barberio, Presidents and political scandal: managing the scandal in the modern era, New York, Palgrave, 2020, page 41. Traduction de l'auteur. 
2. <https://supreme.justia.com/cases/federal/us/506/224/>

3. < https://supreme.justia.com/cases/federal/us/420/35/ >

4. < https://www.washingtonpost.com/wp-srv/national/longterm/watergate/articles/ 102173-2.htm >

5. < https://www.law.cornell.edu/cfr/text/28/600.7 >

6. < https://www.nytimes.com/2017/12/28/us/politics/trump-interview-mueller-russia-chinanorth-korea.html >

7. < https://www.nytimes.com/2018/05/31/us/politics/fact-check-trump-fire-comey-russia.html

8. < https://www.washingtonpost.com/politics/2020/03/04/an-emboldened-trump-says-quietpart-out-loud-about-why-he-fired-jeff-sessions/ >

9. < https://www.justice.gov/ag/page/file/1147981/download >

10. $<$ https://www.justice.gov/storage/report.pdf >

11. < https://www.justice.gov/file/19351/download >

12. < https://int.nyt.com/data/documenthelper/1831-whistleblower-letters/ 33dcb0b8037e9f88ac98/optimized/full.pdf >

13. < https://www.congress.gov/110/plaws/publ409/PLAW-110publ409.pdf >

14. Voir Koncewicz Michael, They Said No to Nixon : Republicans Who Stood Up to the Presidents's Abuses of Power, Los Angeles, University of California Press, 2018.

15. < https://www.politico.com/f/?id=0000016f-aee6-dda2-af6f-efef31580000 >

\section{AUTEUR}

\section{CORENTIN SELLIN}

Corentin Sellin est professeur agrégé d'histoire en CPGE depuis 2012. Il chronique la présidentielle américaine pour le site LesJours.fr. Il est également l'auteur d'un Potomac Paper sur Trump et le parti républicain en 2020 pour l'IFRI (Institut français des Relations Internationales). 